Esta revista forma parte del acervo de la Biblioteca Jurídica Virtual del Instituto de Investigaciones Jurídicas de la UNAM

\title{
Análise dos benefícios sociais da bitcoin como moeda
}

\section{The Social Benefits of the Bitcoin as Currency}

\section{Salete Oro BOFF*}

\section{Natasha AlVes FerReIRA**}

SUMÁRIO: I. Considerações iniciais. II. O que é e como funciona a bitcoin. III. Os desafios da regulamentação da moeda eletrônica. IV. Os benefícios sociais da bitcoin. V. Conclusão. VI. Referências.

* Pós-Doutora em Direito-UFSC. Doutora em Direito UNISINOS. Professora do Programa de Pós-Graduação Stricto Sensu em Direito - Mestrado da IMED. Linha de pesquisa "Mecanismos de efetivação da democracia e da sustentabilidade". Coordenadora do Grupo de Estudos em Desenvolvimento, Inovação e Propriedade Intelectual - GEDIPI.

** Mestranda em Direito pela IMED - Faculdade Meridional. Bolsista de Mestrado da CAPES. Especialista em Direito Público pela Pontifícia Universidade Católica do Rio Grande do Sul-PUCRS.E-mail:naf.natasha@gmail.com.

Artículo recibido el 22 de junio de 2015 Aprobado para publicación el 31 de agosto de 2015 
RESUMO: A tecnologia trouxe conceitos que, fundidos com o campo econômico, permitiram soluções para problemas que a sociedade enfrentou diversas vezes ao longo da sua história, como: inflação, ciclos econômicos, instituições financeiras não confiáveis e a falta de universalização de serviços financeiros a toda a população. Este artigo visa elucidar tanto a parte conceitual da Bitcoin abordando os aspectos históricos, econômicos e jurídicos como também os possíveis benefícios sociais que a moeda pode trazer como uma primeira alternativa, baseada em tecnologia, ao dinheiro tradicional controlado pelo Estado. O método utilizado para tanto é o dedutivo e a técnica de pesquisa é a bibliográfica, partindo-se de exemplos da utilização do dinheiro ao longo da história e da ascensão da Bitcoin como moeda.

Palavras-chave: Bitcoin, Moeda digital, Novas tecnologias, Direito, Benefícios sociais.

ABSTRACT: Technology have brought about concepts which once merged with the economic field have allowed for solutions to be found which society faced several times, such as: inflation, economic cycles, untrustworthy financial institutions and the lack of universalization of financial services for the entire population. This article aims to elucidate both the conceptual part of the Bitcoin addressing the historical, economic and legal aspects as well as the possible social benefits that the currency can bring as a first alternative, based on technology, to the traditional money controlled by the State. The method used for this purpose is the deductive one and the technique is the bibliographical research, starting from the examples of the use of money throughout history and the rise of Bitcoin as currency.

Key words: Bitcoin, Digital currency, New Technologies, Law, Social Benefits.

RÉSUMÉ: La technologie a apporté concepts que, fusionnés avec le domaine économique, ont permis des solutions à des problèmes auxquels la société a fait face à plusieurs reprises tout au long de son histoire, comme l'inflation, les cycles économiques, les institutions financières peu fiables et le manque de services financiers universels à toute la population. Cet article vise à élucider à la fois la partie conceptuelle du Bitcoin abordant les aspects historiques, économiques et juridiques, ainsi que les avantages sociaux potentiels que l'argent peut apporter comme première alternative, basée sur la technologie, à l'argent traditionnel contrôlé par l'Etat. La méthode utilisée pour cela a rapport à la déduction et la technique de recherche est la bibliographique, à commencer par des exemples de l'utilisation de l'argent à travers l'histoire et la montée de Bitcoin comme monnaie.

Mots-clés: Bitcoin, Monnaie numérique, Nouvelles technologies, Droit, Avantages sociaux. 


\section{CONSIDERAÇÕES INICIAIS}

A tecnologia sempre teve um papel fundamental na melhoria da qualidade de vida dos seres humanos. Desde o início da interação do homem com a natureza para prover recursos para o sustento da sua família e comunidade, a inovação foi a propulsora de todos os saltos de qualidade de vida identificados na história. A descoberta de técnicas para plantação extensiva de alimentos permitiu que o homem abandonasse o nomadismo para se fixar em locais ricos em recursos, garantindo mais tempo para se dedicar a outras atividades. A Revolução Industrial possibilitou o acesso a produtos e serviços, antes indisponíveis à maior parte da população, a um número muito maior de pessoas, baseado na melhoria dos processos de produção e na consequente redução dos preços por meio do aumento da escala e criação de novos mercados. Além do aumento da renda per capita, resultou na explosão populacional causada pelo aumento da quantidade de bens e serviços disponíveis. As inovações tecnológicas também influenciaram a forma como as pessoas se comunicam, partindo de redes de relacionamento locais até o fim das fronteiras para a comunicação, independente da sua localização.

Embora muitos autores e até mesmo parte da sociedade ainda enxerguem a tecnologia como algo assustador, baseados nas grandes incertezas que ela traz, ao modificar de modo drástico a sociedade, é inegável perceber que ao olhar para o passado se verificou que ela possibilitou inúmeros avanços para a melhoria das condições de vida da população. Certamente os empreendedores que administravam o negócio de transporte de pessoas em carruagens, no início do século XX, não ficaram satisfeitos quando o carro ficou acessível como veículo de transporte pessoal de massa. Porém, a maior parte da população acaba se beneficiando com o aprimoramento da tecnologia, pois ela, invariavelmente, por processos de tentativa e erro, acaba por produzir resultados melhores, mais rápidos ou mais baratos do que com as técnicas utilizadas anteriormente.

Como resultado do avanço tecnológico tem-se o surgimento da Internet. Desde a sua invenção como uma rede de comunicação que acabou com as fronteiras físicas do conhecimento e da interlocução entre a população de todos os cantos do globo, promoveu uma transformação na forma de comunicação das pessoas. 
Esta revista forma parte del acervo de la Biblioteca Jurídica Virtual del Instituto de Investigaciones Jurídicas de la UNAM

O compartilhamento de informações cresceu de forma tão rápida que a troca de dados entre dois pontos já não é suficiente para alimentar o ímpeto de consumo de informações da nossa sociedade. O desenvolvimento de tecnologias 'peer-to-peer', baseadas no compartilhamento descentralizado de informações, ampliou os horizontes da comunicação. Por meio desta tecnologia, a troca de dados não se dá a partir do ponto $\mathrm{A}$ ao ponto $\mathrm{B}$, mas sim entre todos os participantes de uma comunidade virtual. Ou seja, cada usuário da rede tem um fragmento da informação, permitindo que ela funcione sem que todos os pontos estejam ligados entre si simultaneamente. Ao mesmo tempo em que todos detêm a informação, ninguém realmente a possui. Essa é a tecnologia que deu origem à Bitcoin, uma moeda baseada em uma rede 'peer-to-peer', sem controle central, criptografada, com regras definidas por programação e que não pode ter sua base monetária, quantidade de dinheiro disponível, adulterada.

Apesar da sociedade ter experimentado inovações na maior parte dos campos da ciência e da própria economia, o dinheiro, desde a sua invenção, não sofreu muitas disrupturas para alterar a forma como as pessoas o utilizam. O arranjo de controle governamental sobre a sua utilização, tanto para garantir autenticidade como para padronização do meio circulante, teve papel fundamental para o início do desenvolvimento do comércio e se consolidou como paradigma até os dias de hoje. Friedrich Hayek, ${ }^{2}$ ganhador do prêmio Nobel de economia em 1974, corrobora essa afirmativa, porém levantando a problemática dos monopólios, como o do dinheiro:

Quando a economia monetária ainda estava expandindo-se lentamente nas regiões mais remotas e um dos principais problemas era ensinar a numerosos indivíduos a arte de fazer cálculos em dinheiro (o que não se deu há tanto tempo assim), nessa época, talvez, um único tipo de dinheiro, de fácil identificação, possa ter sido de considerável valia. E pode-se argumentar que o uso exclusivo de um único tipo uniforme de dinheiro auxiliou grandemente a comparação de preços, e, portanto, o crescimento da competição e o mercado. Do mesmo modo, quando a autenticidade do dinheiro metálico só podia ser comprovada através de um difícil processo

1 Arquitetura de redes onde cada um dos pontos de comunicação funciona ao mesmo tempo como cliente e como servidor, possibilitando o compartilhamento de informações ou dados sem a necessidade de um servidor ou nodo central.

2 Hayek, Friedrich A., Desestatização do Dinheiro, São Paulo, Instituto Ludwig Von Mises, Brasil, 2011. pp. 30 e 31. 
Esta revista forma parte del acervo de la Biblioteca Jurídica Virtual del Instituto de Investigaciones Jurídicas de la UNAM

de quilatação, para o qual a pessoa comum não dispunha nem da habilidade nem do equipamento necessários, era possível argumentar com segurança em favor de se garantir a pureza das moedas com a marca de uma autoridade amplamente reconhecida que, fora dos grandes centros comerciais, só poderia ser o governo. Mas, hoje, essas vantagens iniciais, que poderiam ter servido de desculpa para que os governos se apropriassem do direito exclusivo de emitir dinheiro metálico, certamente não têm um peso maior do que o das desvantagens desse sistema. Seus defeitos são os mesmos dos de todos os monopólios: somos obrigados a consumir seus produtos mesmo que sejam insatisfatórios e, acima de tudo, tal sistema impede a descoberta de melhores métodos de satisfazer necessidades que não digam respeito ou não interessem ao monopolista.

A partir disso, a Bitcoin, considerada por alguns a maior inovação financeira dos últimos anos, poderia resolver problemas monetários enfrentados pela sociedade, desde a criação do dinheiro, como a inflação, a falta de acesso a serviços financeiros em regiões remotas e os altos custos para transações. Estes problemas afetam principalmente a população mais pobre. A popularização de uma moeda eletrônica, baseada em uma rede descentralizada e sem um controle central, teoricamente, possibilitaria a diminuição desses problemas.

O presente artigo objetiva elucidar tanto a parte conceitual da Bitcoin abordando os aspectos históricos, econômicos e jurídicos como também os possíveis benefícios sociais que a moeda pode trazer como uma primeira alternativa, baseada em tecnologia, ao dinheiro tradicional controlado pelo Estado.

\section{O QUE É E COMO FUNCIONA A BITCOIN}

O embrião da Bitcoin se deu em 2009, com a publicação de um paper ${ }^{3}$ formulado por um autor, ou grupo de autores, desconhecido sob o pseudônimo de Satoshi Nakamoto. Neste artigo, o autor introduziu a ideia de que a tecnologia 'peer-to-peer' poderia ser a base de um sistema de dinheiro

3 Nakamoto, Satoshi, Bitcoin: A Peer-to-Peer Electronic Cash System. Disponível em: https: / / bitcoin.org/bitcoin.pdf. Acesso em 10,Jan. 2015. 
Esta revista forma parte del acervo de la Biblioteca Jurídica Virtual del Instituto de Investigaciones Jurídicas de la UNAM

eletrônico, por meio da qual duas partes realizariam uma transação sem a necessidade de uma terceira e confiável envolvida, como instituições financeiras. Conforme a ideia do autor, ${ }^{4}$ a necessidade de transações reversíveis, do modelo atual, aumenta os custos de qualquer transação eletrônica, pois exige uma instituição financeira confiável para a realização dos acordos.

Commerce on the Internet has come to rely almost exclusively on financial institutions serving as trusted third parties to process electronic payments. While the system works well enough for most transactions, it still suffers from the inherent weaknesses of the trust based model. Completely non-reversible transactions are not really possible, since financial institutions cannot avoid mediating disputes. The cost of mediation increases transaction costs, limiting the minimum practical transaction size and cutting off the possibility for small casual transactions, and there is a broader cost in the loss of ability to make non-reversible payments for nonreversible services. With the possibility of reversal, the need for trust spreads. Merchants must be wary of their customers, hassling them for more information than they would otherwise need. A certain percentage of fraud is accepted as unavoidable. These costs and payment uncertainties can be avoided in person by using physical currency, but no mechanism exists to make payments over a communications channel without a trusted party. ${ }^{5}$

A Bitcoin propõe um sistema de transações eletrônicas que não depende de confiança. Por meio de um esquema de assinaturas digitais (semelhante ao atual) funciona via uma rede 'peer-to-peer', no qual todo esse ecossiste-

4 Ibidem, p. 1.

5 O comércio na Internet tem vindo a depender quase exclusivamente de instituições financeiras que servem como "terceiros" de confiança para processar pagamentos eletrônicos. Enquanto o sistema funciona bem o suficiente para a maioria das operações, que ainda sofrem com as deficiências inerentes ao modelo baseado em confiança. As transações completamente não reversíveis não são realmente possíveis, uma vez que as instituições financeiras não podem evitar a mediação de disputas. O custo da mediação aumenta os custos de transação, limitando o tamanho mínimo da transação prática e cortando a possibilidade de pequenas transações ocasionais. Há ainda um custo mais amplo na perda da capacidade de fazer pagamentos não reversíveis para serviços não reversíveis. Com a possibilidade de reversão, a necessidade por confiança aumenta. Os comerciantes devem ser cautelosos com os seus clientes para obter mais informações do que seria realmente necessário de outra forma. Uma certa percentagem de fraude é aceito como inevitável. Estes custos e incertezas de pagamento podem ser evitados pessoalmente usando moeda física, mas não existe nenhum mecanismo para fazer pagamentos ao longo de um canal de comunicações sem uma parte confiável [tradução livre]. 
Esta revista forma parte del acervo de la Biblioteca Jurídica Virtual del Instituto de Investigaciones Jurídicas de la UNAM

ma, conjunto de computadores conectados processando somente pedaços da informação de uma transação, utiliza um software para validar todas as transações. O software é de código aberto e todos os seus usuários podem verificar as regras de funcionamento da moeda, além de desenvolver possíveis melhorias que poderão ser aprovadas e utilizadas pela comunidade. A respeito das propriedades da Bitcoin, ${ }^{6}$ Bayern afirma:

Bitcoins have value at least in part because the software (if it works properly, as it has for the most part done so far) ensures (1) their scarcity (people cannot arbitrarily create Bitcoins; they arise only through the expenditure of real computing power, as judged reliably by the Bitcoin software); (2) their security (transmitting bitcoins requires knowledge of their "secret key", a number that it is statistically impossible to guess); and (3) the ability to transmit them with relative ease. ${ }^{7}$

A segurança do funcionamento do sistema está garantida contanto que mais de cinquenta por cento da rede capaz de produzir capacidade de processamento para validar transações aja de forma honesta. Ou seja, a rede Bitcoin se autorregula, pois o software só é compatível desde que funcione através de um consenso com a utilização das mesmas regras.

Embora pareça um pouco assustador a possibilidade da rede ser controlada por pessoas desonestas, o sistema proposto tende a ser muito mais seguro que o atual, pois fraudes financeiras precisam de uma quantidade muito maior de pessoas para serem executadas se comparadas com o modelo em vigência. Por causa desta característica a Bitcoin é uma rede consensual e que funciona usando um software de código aberto, em que mesmo que possam ser criadas diferentes versões do software os usuários são fortemente incentivados a protegerem esse consenso com a utilização das mesmas regras sempre.

6 Bayern, Shawn, "Dynamic Common Law and Technological Change: The Classification of Bitcoin”, Washington and Lee Law Review Online, vol. 71, Issue 2, 2014. Disponível em: http: / /scholarlycommons. law.wlu.edu/wlulr-online/vol71 / iss1 / 2. Acesso em 16 Jan. 2015. p. 24.

7 Bitcoins têm valor, pelo menos em parte, porque o software (caso ele funciona corretamente, como tem ocorrido em grande parte até o momento) garante (1) sua escassez (as pessoas não podem arbitrariamente criar Bitcoins; eles surgem apenas através do uso de capacidade de computação real, desde que validado confiável pelo software de Bitcoin); (2) a sua segurança (transmissão de bitcoins requer o conhecimento de sua "chave secreta", um número que é estatisticamente impossível adivinhar); e (3) a capacidade de transmití-las (transacioná-las) com relativa facilidade [tradução livre]. 
Esta revista forma parte del acervo de la Biblioteca Jurídica Virtual del Instituto de Investigaciones Jurídicas de la UNAM

Embora seja interessante estudar o complexo funcionamento da Bitcoin, para o usuário comum não é preciso muito conhecimento para realizar transações com a moeda eletrônica. Com o uso de um aplicativo ${ }^{8}$ de celular ou computador, o usuário dispõe de uma carteira que armazena os códigos de acesso às Bitcoins disponíveis, além de autorizar os utilizadores a enviar e receber dinheiro. O software pode ser baixado gratuitamente e o usuário fica responsável por adotar medidas de segurança para o armazenamento da moeda digital, como a adoção de antivírus e processos de backup. Este é um ponto crítico para o uso da Bitcoin, pois necessita que os usuários se preocupem com processos de segurança.

O modelo de certificação digital descentralizada permite transparência em todas as transações, pois elas são armazenadas e divulgadas em uma contabilidade pública denominada blockchain. ${ }^{9}$ Nela são arquivadas todas as transações processadas desde o início da moeda e permite aos computadores de todos os usuários realizarem a verificação da validade de cada transação. Toda a infraestrutura necessária para realizar o processamento das transações é provida pelos próprios usuários de forma sustentável e não precisa de concentração de grandes investimentos individuais para atender à demanda de transações. Esse serviço é chamado de "mineração" e recompensa com Bitcoins os usuários que contribuem para a manutenção deste sistema, confirmando transações a serem realizadas e adicionadas à contabilidade do blockchain. A moeda disponível para este pagamento é originada do aumento da base monetária, de forma constante e estável, programado em seu algoritmo para não exceder o limite de 21 milhões de Bitcoin. Por ter uma quantidade de dinheiro previamente definida a Bitcoin é considerada não inflacionária, pois não é afetada por uma autoridade central com poderes para criar uma grande quantidade de dinheiro 'novo' para financiar seus gastos ou dívidas. Além disso, por suas características que limitam o seu crescimento infinito, simula artificialmente a escassez, sendo muitas vezes comparada ao ouro por causa disso. ${ }^{10}$

8 Frequently Asked Questions. Disponível em: https://bitcoin.org/en/faq. Acesso em 12 Jan. 2015.

9 How does Bitcoin work?. Disponível em: https://bitcoin.org/en/how-it-works. Acesso em 15 Jan. 2015.

10 Lo, Stephanie e Wang, J. Christina, Bitcoin as Money? Current Policy Perspectives, Boston, Federal Reserve Bank of Boston, no. 14-4. 4 Set. 2014. Disponível em: http: / / coin-turk.com / wp-content/uploads/2014/09/Bitcoin-as-Money.pdf. Acesso em 25 Jan. 2015. p.11. 
Esta revista forma parte del acervo de la Biblioteca Jurídica Virtual del Instituto de Investigaciones Jurídicas de la UNAM

Para entender melhor como a tecnologia da rede Bitcoin é um avanço em relação ao sistema financeiro atual, tanto em relação à simplificação de processos como em redução de custos, é possível analisar o exemplo ${ }^{11}$ de uma compra em uma cafeteria. Se um consumidor compra com um cartão de crédito o processo parece bastante simples: passar o cartão, colocar a senha ou assinatura e pegar o café e ir embora. Porém, por trás desta transação existem diversas instituições como: o processador de faturamento, a companhia de cartão de crédito (Visa, Mastercard, etc.), o banco do consumidor, o banco do proprietário da cafeteria e o sistema de pagamentos gerido pelo Banco Central, todos eles compartilhando as informações bancárias do comprador. Caso não ocorra nenhum problema e a transação seja aprovada, o proprietário do café paga uma taxa de $2 \%$ a $5 \%$ do valor da transação e recebe o dinheiro na sua conta bancária alguns dias depois, dependendo do país em que a cafeteria se encontra. Já para as compras em Bitcoin é necessário que o estabelecimento aceite este meio de pagamento (hoje mais de 82 mil comerciantes aceitam a moeda eletrônica em todo o mundo) e que o usuário abra o aplicativo que contém a sua carteira de Bitcoins no seu smartphone. Para que a compra se concretize o comprador apresenta o $Q R$ Code, ${ }^{12}$ que contém uma senha incorporada para desbloquear o endereço das suas Bitcoins, ao dono do estabelecimento. A partir de um dispositivo de leitura de $Q R$ Code é informada publicamente a rede Bitcoins a transferência do valor relativo à compra do café. A transação é imediatamente transmitida para a rede, via dados alfanuméricos, e não pode ser atribuída a nenhuma pessoa, o que garante a privacidade da transação. $\mathrm{O}$ processamento da transferência é realizado pelos "mineradores" a cada 10 minutos. O computador da rede que realizou a transação acrescenta os dados da transferência na contabilidade da blockchain, que solicita que os computadores de todos os outros minerados investiguem a validade da operação. Uma vez que a transação é considerada legítima pelos computadores da rede, em um período entre 10 minutos e 1 hora, as Bitcoins são formalmente transferidas para a carteira do proprietário do café. Isso representa

11 Casey, Michael J. e Vigna, Paul, "Bitcoin and the Digital-Currency Revolution”, The Wall Street Journal, 23 Jan. 2015. Disponível em: http: / /www.wsj.com/articles/the-revolutionarypower-of-digital-currency-1422035061. Acesso em 24,Jan.2015.

12 Código de barras bidimensional utilizado para transmissão de informações via dispositivos móveis, a partir de uma câmera que detecta as informações a serem interpretadas por um software. É geralmente utilizado no gerenciamento de estoque em indústria e comércio. 
Esta revista forma parte del acervo de la Biblioteca Jurídica Virtual del Instituto de Investigaciones Jurídicas de la UNAM

uma grande diferença de tempo se for considerado o período de dois a três dias para o processo completo de uma transferência comum. Além disso, a transação de Bitcoins praticamente não tem custo algum.

\section{OS DESAFIOS DA REGULAMENTAÇÃO DA MOEDA ELETRÔNICA}

A sociedade reflete muito dos seus anseios por meio dos rumos da tecnologia. A resolução de problemas sob a forma de inovação produziu resultados fantásticos em muitas áreas do conhecimento, porém, este processo nunca foi linear, pois vários avanços foram retardados por pressão de grupos que não tinham interesse em modificar a sociedade em alguns aspectos.

Recentemente, o aplicativo de transporte executivo chamado UBER foi proibido em alguns países, como Espanha ${ }^{13}$ e Alemanha. ${ }^{14}$ De acordo com a justificativa da proibição, os motoristas que trabalhavam pelo sistema do UBER exerciam concorrência desleal sobre os taxistas por não estarem sob a mesma regulação que eles. Apesar de esta justificativa fazer sentido pela perspectiva dos taxistas, o aplicativo foi criado com a intenção de resolver um problema que é o mal serviço prestado por muitos taxistas, que muitas vezes circulam em número insuficiente em relação a demanda de muitas metrópoles. A empresa UBER percebeu uma oportunidade de negócios a partir desta demanda latente e criou um aplicativo de caronas pagas, e que mais tarde ampliou seus serviços para um sistema de transporte executivo. Em contraponto a isso, os grupos dos taxistas se organizaram, em diversas partes do mundo, para fazer lobby a fim de garantir a proibição do aplicativo. ${ }^{15}$ Apesar da regulação do setor de transporte ter sido criada, com a melhor das intenções, para garantir a segurança e um bom serviço prestado à população, muitas pessoas preferem alternativas para a regulação do Estado, como no caso do UBER.

13 Justiça alemã proíbe utilização de polémico serviço Uber. 2, Set.2014. Disponível em: http: / / www.tsf.pt/PaginaInicial/Vida/Interior.aspx?content_id=4104672. Acesso em 15 Jan. 2015.

14 Espanha proíbe uso do aplicativo Uber. 9 Dez. 2014. Disponível em: http://tecnologia.ig. com.br/2014-12-09/espanha-proibe-uso-do-aplicativo-uber.html. Acesso em 16 Jan. 2015.

15 Thomson, Amy. Taxistas de Londres planejam protesto contra o app Uber. 9 Mai. 2014. Disponível em: http://exame.abril.com.br/tecnologia/noticias/taxistas-de-londres-planejam-protestocontra-o-app-uber. Acesso em 22 Jan. 2015. 
A sociedade pode encontrar soluções tão ou mais eficientes do que a regulação governamental. A aplicação de rankings de reputação tem sido amplamente utilizada pela população com o objetivo de ter maior segurança para a contratação de serviços e produtos pela internet. No caso do UBER, um motorista tem a motivação de conquistar boas reputações para continuar a prestar serviços, sendo ele um profissional ou alguém que oferece serviços eventualmente para ampliar a sua renda em períodos de desemprego ou folga do trabalho.

Outro exemplo semelhante é o aplicativo AIR BNB, um marketplace ${ }^{16}$ que reúne pessoas que querem colocar suas casas ou apartamentos para alugar a interessados por hospedagens mais baratas e flexíveis. Apesar de não regulado pelo Estado, o aplicativo funciona muito bem com um sistema de reputação e comentários. Além de ler sobre a experiência de outras pessoas que, por exemplo, alugaram um estúdio em uma cidade na Europa, é possível também descobrir se o proprietário é confiável ou não, baseado nas críticas públicas. O AIR BNB só realiza pagamentos aos proprietários após o final da estada do hóspede, garantindo que seja prestado o melhor serviço possível. Neste modelo de negócio os proprietários que prestam serviços ruins e enganam seus consumidores são rapidamente expulsos do mercado. Da mesma forma, os maus hóspedes podem ser recusados por não apresentarem bom comportamento, diminuindo assim eventuais riscos para os proprietários. Nos exemplos citados, existe uma motivação dupla: tanto para garantir a credibilidade do aplicativo, beneficiando seus usuários, como para a manutenção dos acordos para que locatários e proprietárias façam acordos benéficos para ambas às partes.

São inúmeros os exemplos em que a tecnologia contribuiu para a resolução de problemas gerados pela regulação estatal ou por sua ausência. A sociedade não determina a tecnologia, esse processo é orgânico e aleatório por meio de processos de tentativa e erro. No entanto, ela pode atrasar o seu desenvolvimento a partir de legislações que permitam a intervenção estatal.

De acordo com Pérez Luño, o exercício de valores democráticos e de direitos humanos representa uma sociedade onde as Novas Tecnologias (NT) e as Tecnologias da Informação e da Comunicação (TIC), especialmente a rede, são a representação da cultura da sociedade. A "sociedade da infor-

16 Espaço virtual destinado a realização de comércio eletrônico de produtos ou serviços. 
Esta revista forma parte del acervo de la Biblioteca Jurídica Virtual del Instituto de Investigaciones Jurídicas de la UNAM www.juridicas.unam.mx

mação" e a "era da Internet" 17 são expressões utilizadas para descrever o momento atual vivido pela sociedade. A sociedade da informação tem tanto acesso ao conhecimento que produz inovações a um ritmo difícil de ser acompanhado pela legislação e que evidencia, em tempo real, as transformações vividas pela sociedade.

A partir do grande crescimento que a Bitcoin teve, principalmente nos últimos dois anos, os governantes e legisladores têm estudado a moeda tanto para entendê-la como para tentar aplicar ou criar regulações para garantir um funcionamento que não coloque em risco seus usuários ou mesmo o status quo do modelo de sistema financeiro atual. Nesse grupo estão tanto pessoas interessadas em melhorar o sistema atual, buscando entender como a tecnologia da Bitcoin pode influenciá-lo positivamente, como pessoas que não enxergam com bons olhos as características revolucionárias da moeda eletrônica.

A maioria dos países ainda não se pronunciou a respeito da legalidade da Bitcoin. Enquanto é considerada legal em países como Estados Unidos, ${ }^{18}$ Austrália ${ }^{19}$ e Alemanha, ${ }^{20}$ a moeda foi banida e é considerada ilegal em países como Bangladesh, ${ }^{21}$ Bolívia, ${ }^{22}$ Equador, ${ }^{23}$ Islândia e Quirguistão. ${ }^{24}$

17 Pérez Luño, Antonio Enrique, Los derechos humanos en la sociedad tecnológica, Madrid, Editorial Universitas, p. 41.

18 Raskin, Max, U.S. Agencies to Say Bitcoins Offer Legitimate Benefits, 18 nov. 2013. Disponível em: http://www.bloomberg.com/news/2013-11-18/u-s-agencies-to-say-bitcoins-offer-legitima te-benefits.html. Acesso em 22 Jan. 2015.

19 Southurst, Jon, Australia Will Set Official Tax Guidelines on Bitcoin This Year. 10 Fev. 2014. Disponível em: http: / / www.coindesk.com/australia-official-tax-guidelines-bitcoin-this-year/. Ace-sso em 22 Jan. 2015.

20 Clinch, Mat, Bitcoin recognized by Germany as 'private money', 19 Ago. 2013. Disponível em: http://www.cnbc.com/id/100971898. Acesso em 22 Jan. 2015.

21 Bangladesh warns of jail for Bitcoin traders. 25 Jun. 2011. Disponível em: http: / / business. asiaone.com/news/bangladesh-warns-jail-bitcoin-traders. Acesso em 23 Jan. 2015.

22 Gerencia de entidades financeiras - Prohibicion del uso de monedas y denominaciones monetárias no reguladas em El âmbito del sistema de pagos nacional. 2014. Disponível em: http: / / www.bcb.gob. bo/webdocs/2014/Normativa/Resoluciones/044\%202014.PDF. Acesso em 22 Jan. 2015.

23 Higgins, Stan. Ecuador Bans Bitcoin, Plans Own Digital Money. 25 Jul. 2014. Disponível em: http: / / www.coindesk.com/ecuador-bans-bitcoin-legislative-vote/. Acesso em 23 Jan. 2015.

24 Rizzo, Pete. Kyrgyzstan: Bitcoin Payments Violate State Law. 4 Ago. 2014. Disponível em: http: / / www.coindesk.com/kyrgyzstan-bitcoin-payments-violate-state-law/. Acesso em 23 jan. 2015. 
Esta revista forma parte del acervo de la Biblioteca Jurídica Virtual del Instituto de Investigaciones Jurídicas de la UNAM

No Brasil a Bitcoin não é considerada moeda pelo governo e é classificada como ativo financeiro. De acordo com a Receita Federal, ${ }^{25}$ o investidor que movimenta acima de $\mathrm{R} \$ 35.000,00$ deve pagar uma alíquota de 15\% de imposto de renda sobre o seu ganho de capital. Segundo ${ }^{26}$ o artigo 55, inciso IV do Regulamento, Decreto n³.000/199927 do Imposto de Renda, são também tributáveis "os rendimentos recebidos na forma de bens ou direitos, avaliados em dinheiro, pelo valor que tiverem na data da percepção”.

O cenário mais comum na tentativa de regulação da Bitcoin é a busca por adequação da nova tecnologia às regulamentações existentes aplicadas ao sistema financeiro. Apesar de ser o modo mais simples de tratar a questão, levantam-se diversos problemas quanto a sua classificação, pois a moeda tem características tanto de dinheiro como de ativo financeiro, como ações ou ouro, e de moeda virtual. Para o Banco Central Europeu a Bitcoin é considerada um esquema de moeda virtual, definido e categorizado da seguinte forma: ${ }^{28}$

.... a virtual currency is a type of unregulated, digital money, which is issued and usually controlled by its developers, and used and accepted among the members of a specific virtual community". This definition may need to be adapted in future if fundamental characteristics change.

There are typically two ways to obtain virtual currencies. In many virtual currency schemes, the fastest way is to purchase it using "real" money at a conversion rate that has been previously established; the virtual currency itself usually has no commodity-backed value. Secondly, users can often increase their stock by engaging in specific activities, for instance by responding to a promotion or advertisement or by completing an online survey. ${ }^{29}$

25 Rapoza, Keneth. Brazil Follows IRS, Declares Bitcoin Gains Taxable. 4 Abr. 2014. Disponível em: http://www.forbes.com/sites/kenrapoza/2014/04/07/brazil-follows-irs-declares-bitcoin-gains -taxable/. Acesso em 23 Jan. 2015.

26 Zampieri, Gabriel Carvalho, Natureza jurídica e tributação da bitcoin, 2014. Disponível em: http: / / gczampieri.jusbrasil.com.br/artigos/118688250/natureza-juridica-e-tributacao-da-bit coin. Acesso em 23 Jan. 2015.

27 Decreto no. 3.000, de 26 de março de 1999. Tributação das Pessoas Físicas - ( Livro 1 Art $2^{\circ}$ a 145 ) 26 Mar. 2009. Disponível em: http: / / www.receita.fazenda.gov.br/Legislacao/rir/ Livro1.htm. Acesso em 22,Jan.2015.

28 Virtual Currency Schemes. European Central Bank. Out. 2012. Disponível em: http://www. ecb.europa.eu/pub/pdf/other/virtualcurrencyschemes201210en.pdf. Acesso em 19 Jan. 2015. p. 13.

29 “...uma moeda virtual é um tipo de, o dinheiro digital não regulamentado, que é emitido e, geralmente, controlado por seus desenvolvedores, e utilizado e aceito entre os membros 
Esta revista forma parte del acervo de la Biblioteca Jurídica Virtual del Instituto de Investigaciones Jurídicas de la UNAM

Uma das agências do tesouro dos Estados Unidos, a Financial Crimes Enforcement Network (FinCEN), emitiu um guia sobre as atividades financeiras que envolvam moedas virtuais. De acordo com a FICEN, ${ }^{30}$ a diferença entre a moeda tradicional e a moeda virtual é a seguinte:

FinCEN's regulations define currency (also referred to as "real" currency) as "the coin and paper money of the United States or of any other country that [i] is designated as legal tender and that [ii] circulates and [iii] is customarily used and accepted as a medium of exchange in the country of issuance."3 In contrast to real currency, "virtual" currency is a medium of exchange that operates like a currency in some environments, but does not have all the attributes of real currency. In particular, virtual currency does not have legal tender status in any jurisdiction. This guidance addresses "convertible" virtual currency. This type of virtual currency either has an equivalent value in real currency, or acts as a substitute for real currency. ${ }^{31}$

Segundo o entendimento das leis norte-americanas, não se aplica o direito de propriedade pessoal ${ }^{32}$ às Bitcoins. O que o investidor tem é um contra-

de uma comunidade virtual específica”. Esta definição pode necessitar de ser adaptada no futuro, se as características fundamentais mudarem. Há tipicamente duas maneiras de obter moedas virtuais. Em muitos esquemas de moeda virtual, a maneira mais rápida é comprá-la usando o dinheiro "real" a uma taxa de conversão que foi previamente estabelecida; a própria moeda virtual geralmente não tem valor lastreados em commodity. Em segundo lugar, os usuários muitas vezes podem aumentar o seu estoque por engajar-se em atividades específicas, por exemplo, respondendo a uma promoção ou propaganda ou através do preenchimento de um inquérito online (tradução livre).

30 FIN-2013-G001. Application of FinCEN's Regulations to Persons Administering, Exchanging, or UsingVirtual Currencies. 18,Mar.2013. Disponível em: http: / / fincen.gov/statutes_regs/guidan ce/html/FIN-2013-G001.html. Acesso em 25 Jan. 2015.

31 As regulações FinCEN definem moeda (também referida como moeda "real") como "moeda e papel moeda dos Estados Unidos ou de qualquer outro país que [i] é designado como o encarregado legal e que [ii] circula e [iii] é habitualmente utilizado e aceita como um meio de troca no país de emissão". Em contraste com moeda real a "moeda virtual" é um meio de troca que funciona como uma moeda em alguns ambientes, mas não tem todos os atributos de uma moeda real. Em particular, a moeda virtual não têm curso legal em qualquer jurisdição. Essa orientação aborda moeda virtual “conversível”. Este tipo de moeda virtual ou tem um valor equivalente em moeda real, ou age como um substituto para a moeda real [tradução livre].

32 Bayern, Shawn, "Dynamic Common Law and Technological Change: The Classification of Bitcoin”, Washington and Lee Law Review Online, vol. 71. Issue 2, 2014. Disponível em: http: / /scholarlycommons. law.wlu.edu/wlulr-online/vol71/iss1/2. Acesso em 16 Jan. 2015, p. 26. 
Esta revista forma parte del acervo de la Biblioteca Jurídica Virtual del Instituto de Investigaciones Jurídicas de la UNAM

to direto com o operador do site que executa a transação. Guardar uma Bitcoin em uma Exchange ${ }^{33}$ ou Wallet, ${ }^{34}$ por exemplo, ou outro serviço que zele pelos códigos de uma Bitcoin, é similar a um depósito em um banco. Porém, existem algumas diferenças como a questão da regulamentação. Os bancos são muito regulados e segurados, fornecendo garantias que, na maioria das vezes, mostram-se seguras, embora, em casos pontuais, essas garantias mostraram-se ineficientes. Outra diferença é que o termo depósito não se aplica da mesma forma à Bitcoin, uma vez que a criptomoeda apenas é armazenada em um ambiente virtual que protege seu código de acesso. No caso do dinheiro tradicional os depósitos bancários influenciam a quantidade de crédito disponível no mercado, pois o dinheiro tradicional funciona a partir de um sistema de reservas fracionárias ${ }^{35}$ onde os bancos podem ampliar sua oferta de crédito acima de suas reservas reais.

De acordo com a Constituição americana não é proibida a criação ou utilização de moedas privadas. ${ }^{36} \mathrm{~A}$ constituição proíbe somente a cunhagem de dinheiro (U.S. Const. art I § 10) por parte dos Estados. Para promover economias locais e empreendedores foram desenvolvidas diversas moedas alternativas como a Cascadia Hour Exchange em Portland e a Life Dollar em Bellingham, Washington.

A tentativa de adequação da Bitcoin ao sistema atual é bastante complexa. A atividade de mineração não se aplica ao modelo atual, uma vez que na maioria dos países os Bancos Centrais são responsáveis pela criação e emissão de novo dinheiro. Sob este novo paradigma, que simula a escassez e se assemelha ao ouro, os governos não podem aplicar medidas de controle monetário. Porém, a Bitcoin ainda não afeta de forma significativamente o sistema financeiro para que este se torne um problema urgente a ser resolvido pelos agentes interessados na sua regulamentação. Isso só seria possível em um cenário onde parte significativa das transações fosse

33 Empresas especializadas na compra e venda de moedas.

34 Espécie de "cofre virtual" que visa garantir a preservação de códigos referentes aos Bitcoins. É a preservação destes códigos que garante a propriedade da moeda eletrônica

35 A maior parte do dinheiro, nas economias modernas, é criado pelo sistema de reserva fracionária bancária, empréstimos ou quantative easing. Desde o abandono do padrão ouro, em que o dinheiro depositado deveria ter o seu equivalente guardado em ouro, não é mais exigido pelos órgãos reguladores esta relação de equivalência.

36 Brito, Jerry e Castilho, Andrea, Bitcoin: a Primer for Policymakers, Mercatus Center, George Mason University. Disponível em: http://mercatus.org/sites/default/files/Brito_BitcoinPri mer.pdf. Acesso em 25 Jan. 2015, p. 24. 
Esta revista forma parte del acervo de la Biblioteca Jurídica Virtual del Instituto de Investigaciones Jurídicas de la UNAM

realizada a partir da Bitcoin. Por outro lado, já se percebe um movimento de pessoas que começam a utilizar a Bitcoin ou outras moedas eletrônicas para se proteger da inflação, como no caso da Argentina ${ }^{37}$ que possui uma das maiores comunidades de usuários da moeda.

\section{OS BENEFÍCIOS SOCIAIS DA BITCOIN}

Apesar de estar cercada por muitas incertezas causadas pela grande flutuação do seu valor e de criminosos poderem se apropriar de seus benefícios, como a privacidade, para esconder crimes, a Bitcoin apresenta inovações com potencial para causar grande impacto na melhoria da vida da população. Um dos benefícios sociais potencialmente trazidos pela Bitcoin é a universalização de acesso a serviços financeiros. Atualmente um número estimado pelo Banco Mundial e divulgado pelo relatório WSJ Money Beat ${ }^{38}$ de 2,5 bilhões de pessoas com idade adulta, em todo o mundo, não tem qualquer tipo de acesso a bancos e serviços financeiros, como o acesso a crédito, transferências eletrônicas e cartões de pagamento (débito e crédito). Esse número representa a falha dos governos em fornecer um sistema financeiro regulado pelo Estado que consiga chegar a toda população mundial. Entre as principais causas da exclusão financeira estão: documentação inadequada, regulações onerosas e infraestrutura bancária ineficiente causada pelos altos custos para atender microeconomias como, por exemplo, áreas rurais africanas. Além disso, a população sem acesso a bancos também não pode receber empréstimos bancários, mas não por serem maus pagadores, mas porque não podem comprovar suas reputações e seus ativos.

Ainda segundo o mesmo relatório, ${ }^{39} \mathrm{o}$ número de pessoas sem acesso a serviços financeiros poderia ser reduzido em $90 \%$ a partir de tecnologias de pagamentos via dispositivos móveis:

37 Ulrich, Fernando, Bitcoin: Buenos Aires. Moeda na era digital. Infomoney. 29 Set. 2014. Disponível em: http://www.infomoney.com.br/blogs/moeda-na-era-digital/post/3604349/bitcoin -buenos-aires. Acesso em 28 Jan. 2015.

38 Casey, Michael J. World's 'Unbanked' En Route to Financial Inclusion With Mobile Money. Disponível em: http://blogs.wsj.com/moneybeat/2014/11/05/worlds-unbanked-en-route-to-financi al-inclusion-with-mobile-money/. Acesso em 10 Jan. 2015.

39 Idem. 
We feel that we are on the cusp of a revolution," says Rodger Voorhies, director of the Financial Services for the Poor initiative at The Bill \& Melinda Gates Foundation. "The fact that mobile handsets are so distributed provides the opportunity to radically change the cost of payments.” He cites a McKinsey \& Co. study finding that transaction costs for the poor could be cut by $90 \% .{ }^{40}$

As populações sem acesso a bancos acabam tendo que pagar taxas exorbitantes para a utilização de serviços financeiros e dependem de mecanismos informais para a proteção do seu dinheiro. Entre os mecanismos informais estão, por exemplo, a compra de joias e commodities, ficando seus proprietários à mercê do risco de roubo e fatalidades como a perda e a destruição através de desastres naturais. Outro problema da falta de acesso a serviços financeiros é a limitação de mercado para pequenos produtores de áreas remotas devido às altas taxas para realizar micro transações com outras partes do mundo ${ }^{41}$. Este problema poderia ser facilmente resolvido com a adoção de uma moeda com custos mais baixos para transações e que não dependesse de infraestrutura bancária.

De acordo com o CGAP (Consultive Group to Assist Poor) ${ }^{42}$, pode-se aprender com a revolução do microcrédito que mostra que famílias pobres da economia informal são clientes valiosos. Hoje o tamanho dessa economia gira em torno de $\$ 70$ bilhões, com demanda para uma ampliação ainda maior. O resultado é um sucesso não-governamental sustentável que mostra que as famílias pobres também precisam ter acesso a toda gama de serviços financeiros para gerar renda, construir patrimônio, consumir mais e gerenciar riscos que um modelo limitado de microcrédito não pode fornecer.

O microcrédito ainda não é um benefício direto do Bitcoin e nem está no seu escopo de projeto. Porém, o acesso a serviços financeiros é o pri-

40 "Nós sentimos que estamos à beira de uma revolução", diz Rodger Voorhies, diretor da "Serviços Financeiros para a em favor dos Pobres", na Fundação Bill \& Melinda Gates. "O fato de que telefones celulares são tão distribuídos que proporcionam a oportunidade de mudar radicalmente o custo de pagamentos". Ele cita um estudo da McKinsey \& Co. descobrindo que os custos de transação para os pobres poderiam ser cortados em $90 \%$ (tradução livre).

41 Casey, Michael J. World's 'Unbanked' En Route to Financial InclusionWith Mobile Money. Disponível em: http://blogs.wsj.com/moneybeat/2014/11/05/worlds-unbanked-en-route-to-finan cial-inclusion-with-mobile-money/. Acesso em 10 Jan. 2015.

42 CGAP Advancing financial inclusion to improve the lives of the poor. Disponivel em: http: / / www.cgap.org/topics/financial-inclusion. Acesso em 10 Jan. 2015. 
Esta revista forma parte del acervo de la Biblioteca Jurídica Virtual del Instituto de Investigaciones Jurídicas de la UNAM

meiro passo para que a camada mais pobre da população possa ter acesso a diferentes tipos de produtos para efetuar transações e financiar pequenos empreendimentos. A partir de uma alternativa financeira e eletrônica para o papel moeda, baseada em tecnologia de compartilhamento de informações, não é difícil imaginar inovações para a melhoria dos sistemas de crédito atuais. A tecnologia 'peer-to-peer' poderia ser utilizada para formar grupos de investimentos que fornecessem crédito para a base dos usuários de Bitcoin, por exemplo, de forma anônima e com informações transparentes referentes aos riscos para os investidores, baseadas nos dados de inadimplência e com a impossibilidade técnica para discriminação a qualquer grupo da sociedade. Este tipo de serviço já é fornecido pela empresa brasileira situada em San Francisco BTC Jam, ${ }^{43}$ que conecta investidores e empreendedores que precisam de crédito para ampliar seus negócios. Pelo sistema de crowdfunding, ${ }^{44}$ arrecadação online de fundos, a BTC Jam utiliza a rede 'peer-to-peer' da Bitcoin para criar um sistema de reputação algorítmica que tira as instituições financeiras da função intermediárias.

Outro benefício social da Bitcoin é o menor custo para transações. De acordo com o estudo realizado pelo Congressional Research Service, serviço de pesquisa do Congresso americano, pelo fato de todas as transações serem processadas e validadas por uma rede 'peer-to-peer', via mineradores recompensados, não existe a necessidade de uma terceira parte envolvida para uma transação entre dois usuários. Isso se revela como redução de custos, se comparado com prestadores de serviços que validam transações digitais com dinheiro tradicional, como o Paypal ou provedores de cartão de crédito.

A partir da tecnologia da rede Bitcoin, as transações são processadas quase sem custo algum, pois utilizam a própria rede ${ }^{45}$ de usuários para validar as operações no blockchain. A rede é autossustentável, beneficiando os provedores de infraestrutura de processamento (CPU) com micro frações de Bitcoins por processamento. Cada transação possui um delay que pode

43 BTC Jam. Disponível em: https: / /btcjam.com/. Acesso em 10 Jan. 2015.

44 Financiamento coletivo ou obtenção de capital a partir de múltiplas fontes de financiamento. Utilizado geralmente para levantamento de fundos para causas sociais ou ideias inovadoras.

45 Elwall, Craig K.; Murphy, M. Maureen; Seitzinger, Michael V., Bitcoin: Questions, Answers, and Analysis of Legal Issues. 20 Dez. 2013. Disponível em: http://cdn.panteracapital.com/wpcontent/uploads/beb308be2f90c4b55cb8c88d8271a974-crs-report-on-bitcoin-.pdf. Acesso em 10 Jan. 2015 , p. 5. 
Esta revista forma parte del acervo de la Biblioteca Jurídica Virtual del Instituto de Investigaciones Jurídicas de la UNAM

ser ignorado pagando-se taxas para transações preferenciais. Essa taxa é muito baixa e não precisa ser paga pelos usuários que não têm necessidade de acelerar a transação e podem esperar pelo tempo padrão, em torno de 10 minutos. Em relação à diminuição de custos para transações financeiras, Fernando Ulrich afirma que

...o Bitcoin é uma grande promessa de uma forma de reduzir os custos de transação aos pequenos comerciantes e remessas de dinheiro globais, aliviar a pobreza global pelo facilitado acesso ao capital, proteger indivíduos contra controles de capitais e censura, garantir privacidade financeira a grupos oprimidos e estimular inovação (dentro e acima do protocolo Bitcoin). Por outro lado, a natureza descentralizada do Bitcoin também apresenta oportunidades ao crime. O desafio, então, é desenvolver processos que reduzam as oportunidades para criminalidade enquanto mantêm-se os benefícios que Bitcoin oferece. ${ }^{46}$

Provavelmente o benefício social mais importante da Bitcoin seja a proteção dos seus usuários contra a inflação. ${ }^{47} \mathrm{O}$ termo inflação se refere ao aumento dos preços de bens e serviços. Traz como consequência a queda do poder de compra da população, uma vez que há uma queda no valor da moeda circulante. Um fenômeno inflacionário significa que uma quantidade de unidades monetárias compra uma quantidade de bens ou serviços hoje, porém, no futuro, através da desvalorização da moeda, essa mesma quantidade de dinheiro passa a comprar uma quantidade menor dos mesmos bens e serviços.

Atualmente quem controla a quantidade de dinheiro circulante são os Governos que, por meio dos seus bancos centrais, determinam a quantidade adequada de dinheiro disponível. Apesar da maior parte dos economistas ser favorável ao arranjo atual, existe um histórico muito grande de má gestão por parte de governantes em relação à quantidade de moeda circulante. Essa administração utiliza-se da criação excessiva de dinheiro novo para financiar gastos públicos e resulta no empobrecimento da população que passa a ter um poder de compra menor ou a necessitar de maior

46 Ulrich, Fernando, Bitcoin: a moeda na era digital, São Paulo, Instituto Ludwig Von Mises Brasil, 2014, p. 23.

47 Elwall, Craig K. et al., Bitcoin: Questions, Answers, and Analysis of Legal Issues, 20 Dez. 2013. Disponível em: http://cdn.panteracapital.com/wp-content/uploads/beb308be2f90c4b55cb 8c88d8271a974-crs-report-on-bitcoin-.pdf. Acesso em 10 Jan. 2015, p. 6. 
Esta revista forma parte del acervo de la Biblioteca Jurídica Virtual del Instituto de Investigaciones Jurídicas de la UNAM

quantidade de trabalho para manter o seu padrão de vida. A respeito desse fenômeno, quem mais sofre com isso é a população pobre que não tem conhecimento financeiro ou capital para proteger seu patrimônio contra a inflação por meio de investimentos.

Um exemplo extraordinário de hiperinflação é o Zimbábue, primeiro país no século XXI a sofrer este fenômeno. A hiperinflação do país ${ }^{48}$ destruiu sua economia levando quase toda a sua população para a pobreza extrema e forçando a emigração de milhões de Zimbabueanos. Entre 1997 e 2007 a inflação acumulada foi de 3.800.000.000. ${ }^{49}$ Nesse período o padrão de vida caiu 38\%, na contramão do resto do mundo. A fonte da hiperinflação de Zimbábue foi o Banco Central (Reserve Bank of Zimbabwes's) do país através da criação de dinheiro novo.

No caso da Bitcoin, por ser uma moeda descentralizada e que não possui autoridade central para o controle da sua base monetária, o risco de inflação é menor ou nulo, pois o aumento da quantidade de moeda é constante até um limite previamente definido de 21 milhões de unidades monetárias. A partir desse arranjo, nenhum governo pode utilizar-se do artifício da desvalorização da moeda para financiamento dos gastos públicos, através de um aumento do meio circulante, criação de novo dinheiro, para gastos que excedem o valor arrecadado com impostos. Esse benefício social é favorável à população, principalmente a dos países mais pobres que acabam utilizando esse tipo de recurso para tentar viabilizar crescimento econômico. Porém, como a sociedade não fica mais rica com a criação de dinheiro 'novo', a manutenção do valor da moeda tende a beneficiar a maior parte da população.

Outro benefício social da utilização da Bitcoin como moeda é a proteção do dinheiro da população contra o confisco governamental. Por ser uma moeda em que suas informações são descentralizadas e criptografadas não é possível, pelo menos com a tecnologia atual, ter acesso ao dinheiro das pessoas. Desta forma, confisco da poupança, como aconteceu no Brasil nos anos 90, não seria possível. Embora seja uma estratégia governamental muito drástica e impopular ela já aconteceu muitas vezes. Um dos motivos dessa estratégia não ser mais utilizada com tanta frequência é porque os governantes descobriram que utilizar os Bancos Centrais para criar dinhei-

48 Hanke, Steve H. Zimbabwe From Hiperinflation to Growth. Cato Institute, no. 6. Disponível em: http://www.cato.org/pub_display.php?pub_id=9484. Acesso em 17 Jan. 2015, p. 2.

49 Ibidem, p. 1. 
Esta revista forma parte del acervo de la Biblioteca Jurídica Virtual del Instituto de Investigaciones Jurídicas de la UNAM

ro 'novo' é menos impopular, pois seus efeitos são mais suaves e demoram mais tempo até serem percebidos. Os efeitos da criação de dinheiro 'novo' é sempre inflação, pois acontece um aumento do dinheiro disponível sem que haja um aumento de bens e serviços. Embora a população não perca o dinheiro imediatamente, seu poder de compra é diminuído.

Um exemplo recente foi o anúncio de confisco governamental no Chipre em $2013^{50}$ sobre o dinheiro depositado nas contas bancárias. O tamanho do confisco era de 6,75\% em contas com depósitos entre 20 e $100 \mathrm{mil}$ euros e de 9,9\% em contas com depósitos acima de 100 mil euros. Como consequência houve uma corrida da população aos caixas eletrônicos para sacar o dinheiro guardado. A população escolheu diversas formas de proteção alternativa para seu dinheiro. Uma delas foi a compra ${ }^{51}$ massiva de Bitcoins que teve uma valorização grande na época.

Hayek $^{52}$ defendeu que a abolição do monopólio monetário governamental poderia dar lugar à difusão do uso de várias moedas privadas que competiriam entre si e representariam um aperfeiçoamento em relação a esse monopólio. Desta forma, o governo seria privado "não só de um dos principais meios de que se vale para prejudicar a economia e submeter os indivíduos a restrições de sua liberdade, como também de uma das principais causas de sua constante expansão" ${ }^{53}$. De acordo com o autor, é do Estado que a moeda deveria ser protegida, sendo ele apenas responsável pelo controle da autenticidade das moedas.

\section{CONCLUSÃO}

O dinheiro foi uma inovação social que se mostrou um sistema superior ao escambo e permitiu um progresso do comércio e das relações de troca da

50 Correntistas temem crise de confiança na Europa com 'confisco' no Chipre. Disponível em: http: / / www.bbc.co.uk/portuguese/noticias/2013/03/130319_chipre_criseconfianca_ad_ss. Acessado em 16 Jan. 2015.

51 Oliveira, Fabio Machado et al., Bitcoin: o dinheiro com tecnologia de fonte aberta em rede ponto-a-ponto. Disponível em http://www.periodicos.letras.ufmg.br/index.php/anais_linguagem_ tecnologia/article/view/5879. Acessado em 16 Jan. 2015.

52 Hayek, Friedrich A., Direito, Legislação E Liberdade, São Paulo, Visão, 1985, vol. III A Ordem Política de um Povo Livre, p. 62.

53 Ibidem, p. 62. 
Esta revista forma parte del acervo de la Biblioteca Jurídica Virtual del Instituto de Investigaciones Jurídicas de la UNAM

sociedade. Ao longo dos tempos, demonstrou capacidade de transformação para se adaptar a diferentes realidades. O desenvolvimento da internet, decorrente da evolução das tecnologias da informação e da comunicação, permitiu que paradigmas conceituais a respeito do dinheiro fossem quebrados pela sociedade.

A Bitcoin, apesar de estar cercada por muitas incertezas, principalmente por apresentar-se como uma tecnologia nova, ainda desconhecida para a maioria da população e com um sistema baseado em criptografia e algoritmos, o que a torna uma moeda de difícil compreensão, traz inovações que geram um grande impacto na forma de se utilizar o dinheiro. Embora traga muitos pontos de interrogação em relação à sua reputação como a possibilidade de lavagem de dinheiro ou a utilização da moeda por criminosos que querem apagar seus rastros, ela certamente traz benefícios sociais, como a possibilidade de universalização de serviços financeiros, a proteção dos seus usuários contra a inflação e o confisco governamental e a redução de custos em transações financeiras. Além disso, o conceito da moeda eletrônica traz ideias para a melhoria do sistema financeiro atual.

Com o amadurecimento da Bitcoin, ocorrerá uma provável regulamentação que a impactará ou para seu fortalecimento ou para sua decadência. Apesar de ser a moeda eletrônica mais conhecida, não existem garantias de que ela será utilizada por muito tempo nem de que será a criptomoeda, ou moeda eletrônica, definitiva. Como em qualquer outra inovação, existe a possibilidade de defasagem devido ao surgimento de outras moedas que utilizem outro tipo de tecnologia e que sejam até mesmo mais inovadoras que a Bitcoin.

De qualquer forma, é necessário que os legisladores de todo mundo avaliem os possíveis benefícios sociais que podem impactar positivamente a sociedade para evitar a criação de regulações que induzam ao enfraquecimento da moeda. Mesmo que a utilização da Bitcoin possa ser considerada uma forma de contestação ao poder do Estado, ela traz inovações, como o blockchain, com potencial para gerar aprimoramentos de governança, baseados em tecnologia, e que poderiam melhorar até mesmo a relação de transparência entre o Estado e a sociedade. 


\section{REFERÊNCIAS}

Bangladesh warns of jail for Bitcoin traders. 25 Jun. 2011. Disponível em: http: / / business.asiaone.com/news / bangladesh-warns-jail-bitcoin-traders. Acesso em 23 Jan. 2015.

BAyern, Shawn, "Dynamic Common Law and Technological Change: The Classification of Bitcoin", Washington and Lee Law Review Online, vol. 71. Issue 2, 2014. Disponível em: http: / /scholarlycommons.law.wlu.edu/wlulronline/vol71/iss 1/2. Acesso em 16 Jan. 2015.

Brito, Jerry e CASTilHo, Andrea, Bitcoin: a Primer for Policymakers. Mercatus Center, George Mason University. Disponível em: http://mercatus.org/ sites/default/files/Brito_BitcoinPrimer.pdf. Acesso em 25 Jan. 2015.

BTC Jam. Disponível em: https: / /btcjam.com/. Acesso em 10 Jan. 2015.

CASEY, Michael J. e Vigna, Paul, "Bitcoin and the Digital-Currency Revolution”, TheWall Street Journal, 23 Jan. 2015. Disponível em: http: / / www. wsj.com/articles / the-revolutionary-power-of-digital-currency-1422035061. Acesso em 24 Jan. 2015.

CASEY, Michael J., World's “Unbanked”, en Route to Financial InclusionWith Mobile Money. Disponível em: http: / /blogs.wsj.com/moneybeat/2014/ 11 /05 / worlds-unbanked-en-route-to-financial-inclusion-with-mobile-money/. Acesso em 10 Jan. 2015.

CGAP Advancing financial inclusion to improve the lives of the poor. Disponível em: http://www.cgap.org/topics/financial-inclusion. Acesso em 10,Jan. 2015.

Clinch, Mat, Bitcoin recognized by Germany as 'private money', 19 Ago. 2013. Disponível em: http://www.cnbc.com/id/100971898. Acesso em 22,Jan. 2015.

Correntistas temem crise de confiança na Europa com 'confisco' no Chipre. Disponível em: http://www.bbc.co.uk/portuguese/noticias/2013/03/130319_ chipre_criseconfianca_ad_ss. Acessado em 16 Jan. 2015.

Decreto no. 3.000, de 26 de março de 1999. Tributação das Pessoas Físicas - (Livro 1 - Art $2^{\circ}$ a 145) 26 Mar. 2009. Disponível em: http: / / www. receita.fazenda.gov.br/Legislacao/rir/Livro1.htm. Acesso em 22 Jan. 2015.

ElWall, Craig K. et al., Bitcoin: Questions, Answers, and Analysis of Legal Issues, 20 Dez. 2013. Disponível em: http://cdn.panteracapital.com/wp-content / 
Esta revista forma parte del acervo de la Biblioteca Jurídica Virtual del Instituto de Investigaciones Jurídicas de la UNAM

uploads/beb308be2f90c4b55cb8c88d8271 a974-crs-report-on-bitcoin-.pdf. Acesso em 10 Jan. 2015.

Espanha proíbe uso do aplicativo Uber. 9 Dez. 2014. Disponível em: http:// tecnologia.ig.com.br/2014-12-09/espanha-proibe-uso-do-aplicativo-uber. html. Acesso em 16 Jan. 2015.

Frequently Asked Questions. Disponível em: https: / / bitcoin.org/en/faq. Acesso em 12 Jan. 2015.

FIN-2013-G001. Application of FinCEN's Regulations to Persons Administering, Exchanging, or Using Virtual Currencies. 18 Mar. 2013. Disponível em: http: / / fincen.gov/statutes_regs/guidance/html/FIN-2013-G001.html. Acesso em 25,Jan.2015.

Friedman, Milton, Milton Friedman predicts the rise of Bitcoin in 1999! Coin Republic, 30 Ago. 2013. Disponível em: http://www.youtube.com/ watch $_{v}=6$ MnQJFEVY7s. Acesso em 25 Jan. 2015.

Gerencia de entidades financeiras - Prohibicion del uso de monedas y denominaciones monetárias no reguladas em El âmbito del sistema de pagos nacional. 2014. Disponível em: http://www.bcb.gob.bo/webdocs/2014/Normativa/Resolu ciones /044\%202014.PDF. Acesso em 22,Jan.2015.

Hanke, Steve H., Zimbabwe From Hiperinflation to Growth, Cato Institute, no. 6. Disponível em: http://www.cato.org/pub_display.php?pub_id $=9484$. Acesso em 17 Jan. 2015.

HAYeK, Friedrich A., Desestatização do Dinheiro, São Paulo, Instituto Ludwig von Mises, Brasil, 2011.

-, Direito, Legislação e Liberdade, São Paulo, Visão, 1985, vol. III A Ordem Política de um Povo Livre.

Higgins, Stan, Ecuador Bans Bitcoin, Plans Own Digital Money, 25 Jul. 2014. Disponível em: http://www.coindesk.com/ecuador-bans-bitcoin-legislativevote/. Acesso em 23 Jan.2015.

How does Bitcoin work?, Disponível em: https://bitcoin.org/en/how-it-works. Acesso em 15,Jan.2015.

Justiça alemã proíbe utilização de polémico serviço Uber. 2,Set.2014. Disponível em: http://www.tsf.pt/PaginaInicial/Vida/Interior.aspx?content_ id $=4104672$. Acesso em 15,Jan.2015.

Lo, Stephanie e Wang, J. Christina, Bitcoin as Money?. Current Policy Perspectives, Boston, Federal Reserve Bank of Boston, no. 14-4, 4 Set. 2014. Disponível em: http://coin-turk.com/wp-content/uploads/2014/09/Bitcoin-as-Money.pdf. Acesso em 25 Jan. 2015. 
Esta revista forma parte del acervo de la Biblioteca Jurídica Virtual del Instituto de Investigaciones Jurídicas de la UNAM

PÉREZ LuÑo, Antonio Enrique, Los derechos humanos em la sociedad tecnológica, Madrid, Editorial Universitas.

Nakamoto, Satoshi, Bitcoin:A Peer-to-Peer Electronic Cash System. Disponível em: https: / / bitcoin.org/bitcoin.pdf. Acessado em 09 Jan. 2015.

OliveIRA, Fabio Machad et al., Bitcoin: o dinheiro com tecnologia de fonte aberta em rede ponto-a-ponto. Disponível em http: / / www.periodicos.letras.ufmg.br /index.php/anais_linguagem_tecnologia/article/view/5879. Acessado em 16 Jan. 2015.

RAPOZA, Keneth, Brazil Follows IRS, Declares Bitcoin Gains Taxable. 4,Abr.2014. Disponível em: http://www.forbes.com/sites/kenrapoza/ 2014/04/07/brazil-follows-irs-declares-bitcoin-gains-taxable/. Acesso em 23,Jan. 2015.

Raskin, Max, U.S. Agencies to Say Bitcoins Offer Legitimate Benefits. 18 Nov. 2013. Disponível em: http://www.bloomberg.com/news/2013-11-18/u-s -agencies-to-say-bitcoins-offer-legitimate-benefits.html. Acesso em 22 Jan. 2015.

Rizzo, Pete, Kyrgyzstan: Bitcoin Payments Violate State Law, 4 Ago. 2014. Disponível em: http://www.coindesk.com/kyrgyzstan-bitcoin-payments-violate -state-law/. Acesso em 23 Jan. 2015.

SouTHurst, Jon, AustraliaWill Set Official Tax Guidelines on Bitcoin This Year. 10 Fev. 2014. Disponível em: http: / / www.coindesk.com/australia-official-tax-guidelines-bitcoin-this-year/. Acesso em 22 Jan. 2015.

Thomson, Amy, Taxistas de Londres planejam protesto contra o app Uber. 9 Mai. 2014. Disponível em: http://exame.abril.com.br/tecnologia/noti cias/taxistas-de-londres-planejam-protesto-contra-o-app-uber. Acesso em 22 Jan. 2015.

UlRICH, Fernando, Bitcoin: a moeda na era digital, São Paulo, Instituto Ludwig Von Mises Brasil, 2014.

, Bitcoin: Buenos Aires. Moeda na era digital, Infomoney, 29 Set. 2014. Disponível em: http: / / www.infomoney.com.br/blogs/moeda-na-era-digital / post/3604349/bitcoin-buenos-aires. Acesso em 28 Jan. 2015.

Virtual Currency Schemes, European Central Bank, Out. 2012. Disponível em: http: //www.ecb.europa.eu/pub/pdf/other/virtualcurrencyschemes 201210 en.pdf. Acesso em 19 Jan. 2015.

ZAMPIERI, Gabriel Carvalho, Natureza jurídica e tributação da Bitcoin, 2014. Disponível em: http://gczampieri.jusbrasil.com.br/artigos/118688250/ natureza-juridica-e-tributacao-da-bitcoin. Acesso em 23 Jan. 2015. 